\title{
La relation complexe entre l'élevage et la forêt en Amazonie brésilienne : une approche par la modélisation multi-agents
}

Pierre Bommel $^{1,2}$
Thierry Bonaudo $^{3}$
Tienne Barbosa $^{4}$
Jonas Bastos da Veiga $^{5}$
Manuela Vieira Pak $^{1}$
Jean-François Tourrand $^{6}$

${ }^{1}$ Cirad UPR Green

Campus international de Baillarguet

TAC-47 / F 34398 Montpellier cedex 5

France

<manuelka79@hotmail.com>

2 Université de Brasília

Faculté de technologie UnB - FT

Campus universitàrio Darcy Ribeiro

Asa Norte

70.910-900

Brasilia

Brésil

<bommel@cirad.fr>

${ }^{3}$ AgroParisTech Élevage et environnement

16, rue Claude Bernard

F-75231 Paris cedex 05

<thierry.bonaudo@agroparistech.fr>

${ }^{4}$ Embrapa Amazônia Oriental

Trav. Dr. Enéas Pinheiro

CEP 66095-100 Caixa Postal

48 Belém Brési

<tiennejo@yahoo.com.br>

${ }^{5}$ IDESP

1461, Rua Municipalidade

CEP: 66050 - 350 Para Belem

Brésil

<jonas.veiga@superig.com.br>

${ }^{6}$ Cirad GIP IFRA

Inra-Cirad Cirad Drs

04, avenue Agropolis

34398 Montpellier cedex 5

France

<jean-francois.tourrand@cirad.fr>

\begin{abstract}
Résumé
En Amazonie, l'élevage bovin contribue à la construction d'un nouveau territoire du fait et en dépit des impacts qu'il a sur un écosystème forestier dont la préservation fait la quasi-unanimité. Il existe en effet un profond fossé entre d'une part des acteurs locaux, pionniers à la recherche de meilleures conditions de vie, pour lesquels la forêt est une simple réserve de fertilité et, d'autre part, la communauté internationale soucieuse des impacts environnementaux de cette déforestation à grande échelle. Nous avons conçu un système multi-agents (SMA) qui permet de rendre compte des stratégies des éleveurs et agriculteurs qui sont les acteurs clés de cette déforestation, et d'en simuler les impacts. Nos résultats éclairent d'un jour nouveau les questions et enjeux relatifs à l'intensification agroécologique et l'élaboration de politiques publiques durables pour le développement des fronts pionniers sur la Transamazonienne. Nos travaux ouvrent de nouvelles perspectives pour le partage d'information entre leurs diverses parties prenantes. Bien que conçu pour représenter des dynamiques locales, le modèle apparaît suffisamment générique pour être appliqué à d'autres situations amazoniennes.
\end{abstract}

Mots clés : Amazonie ; Brésil ; déforestation ; élevage ; modélisation ; système multi-agents.

Thèmes : forêts ; productions animales ; ressources naturelles et environnement ; territoire, foncier, politique agricole et alimentaire.

\section{Abstract}

The complex relationship between cattle ranching and the forest in Brazilian Amazonia. A multiagent modelling approach

In Amazonia, cattle ranching plays a particular role in the construction of new territory, especially because it affects the Amazon Rainforest, the conservation of which represents very high stakes. There is a wide and obvious gap between on the one hand local actors, pioneers seeking better living conditions and for whom the natural forest is perceived as a simple reserve of fertility and, on the other hand, the international community concerned by environmental impact. We describe several versions of a multi-agent model and their results as information sharing between stakeholders as along with new research questions regarding agro-ecological intensification and the drawing up of durable public policies. Although it has been designed to represent local dynamics, this model could probably be used in other Amazonian areas.

Key words: Amazonia; animal breeding; Brazil; deforestation; modelling; multiagent system.

Subjects: animal productions; forests; natural resources and environment; territory, land use, agricultural and food production policy. vec une centaine de millions de têtes de bétail, l'Amazonie brésilienne est devenue un des pre- miers bassins mondiaux de production de viande bovine et, d'ici peu, de produits laitiers. La croissance de son cheptel et le 
fort impact environnemental de l'élevage en font un terrain essentiel pour étudier les relations élevage-environnement, car cette croissance s'accompagne d'une augmentation des surfaces herbagères au détriment de la forêt équatoriale.

Notre objectif est de brosser un tableau général des raisons de la déforestation à partir de la description d'un système multi-agents (SMA) en se focalisant sur les stratégies des acteurs, éleveurs et agriculteurs qui sont les éléments clés de cette déforestation.

Ces travaux s'inscrivent dans le projet Trans ${ }^{1}$ qui vise à comprendre les transformations des élevages et leurs conséquences sur les dynamiques territoriales.

\section{Raisons de l'expansion de l'élevage} en Amazonie

L'Amazonie brésilienne occupe une place à part dans le monde pastoral pour plusieurs raisons. La première est le caractère récent du développement de l'élevage. En 1970, l'Amazonie comptait trois à cinq millions de bovins élevés sur les savanes naturelles des berges de l'Amazone et sur l'île de Marajó. Dans les années 1970, des pionniers venus du Sud du Brésil commencèrent à déforester pour installer leurs troupeaux. Quarante ans plus tard, cette colonisation, soutenue par le gouvernement, a transformé un million de $\mathrm{km}^{2}$ de forêt en pâturage pour alimenter 100 millions de bovins, soit 20 à $25 \%$ de la forêt amazonienne totale.

Une autre raison est la croissance rapide du cheptel (8\% par an), fondée sur une logique d'expansion. Le climat, propice à l'herbe et la culture, fournit une alimentation abondante pour les troupeaux et favorise une bonne productivité numérique et une croissance pondérale rapide des animaux dans les systèmes de production bovins.

La troisième particularité est le lien étroit entre l'expansion de l'élevage et la déforestation. Chaque année, un colon brûle

\footnotetext{
${ }^{1}$ Programme ADD (Agriculture et développement durable), coordonné par Benoit Dedieu, Institut national de la recherche agronomique (Inra). Financement de l'Agence nationale pour la recherche (ANR).
}

une parcelle forestière de son exploitation qu'il plante en cultures vivrières puis en fourrages. En moyenne, 2-3 hectares sont ainsi mis en pâture annuellement par les petits éleveurs et 50-100 hectares dans les ranchs. À l'échelle du bassin, chaque année deux à trois millions d'hectares de forêt sont brûlés depuis 50 ans.

Ainsi, l'Amazonie est devenue un territoire majeur de la production bovine, concourant à faire du Brésil le premier exportateur mondial de viande. En contrepartie, ce territoire est devenu un des grands pollueurs par les gaz à effet de serre issus de la déforestation et de l'élevage, et contribue à l'érosion de la biodiversité du plus riche réservoir forestier de la planète.

Veiga et al. (2004) et Piketty et al. (2005) ont montré que divers facteurs se combinent pour induire un réel engouement des acteurs ruraux pour l'élevage bovin.

\section{Efficacité de la filière bovine et stabilité des prix}

L'efficacité de la filière permet à tout éleveur d'avoir l'assurance de pouvoir vendre ses animaux à un prix indexé sur le marché international. Perçu comme un placement, l'élevage est devenu une valeur refuge lors des périodes d'inflation liée aux crises des pays émergents. Durant ces périodes, le prix du bétail fut, par sa stabilité, la référence monétaire en milieu rural. On estimait le prix d'un bien ou d'un service en nombre de bovins (Poccard Chapuis et al., 2005).

\section{Climat favorable et efficacité des systèmes fourragers}

Les conditions climatiques associant chaleur, humidité et bonne répartition annuelle des pluies favorisent la production fourragère. La fertilité des sols, notamment après brûlis, contribue à la bonne pousse de l'herbe. En outre, le caractère envahissant des herbacées sélectionnées facilite la lutte contre les adventices et le recrû forestier. Enfin, la recherche agronomique qui a accompagné la colonisation a su élaborer des systèmes fourragers performants et résistants à la dégradation des herbages qui caractérisait les premiers itinéraires techniques importés d'Afrique tropicale (Veiga et al., 2004).

\section{Flexibilité du travail en élevage}

L'élevage allaitant à l'herbe en parcelles clôturées exige une attention plus souple que les autres systèmes d'élevage. En dehors des périodes de vêlage, le troupeau peut rester un à trois jours sans surveillance, même si une visite quotidienne est préférable. L'exploitant peut confier cette tâche à un vacher. En termes de quantité de main-d'œuvre, deux personnes suffisent pour garder 250-300 vaches allaitantes et un homme seul à cheval peut surveiller 800-1 000 bouvillons, les tâches annexes telles que l'entretien des pâturages étant souvent assurées par l'achat de main d'œuvre temporaire.

\section{Appui des politiques publiques}

Depuis le début de la colonisation, les pouvoirs publics soutiennent le secteur de l'élevage en Amazonie. Les pionniers reconnaissent tous le rôle majeur des prêts bonifiés accordés par l'État, via le BASA (Banque d'Amazonie) et la Sudam (Institution chargée du développement territorial), dans le développement de l'élevage familial (Toni, 1999). De plus, un réseau routier a été créé pour faciliter la commercialisation du bétail puis celle du lait. Quelle que soit l'orientation politique, le soutien n'a jamais fléchi, même s'il allait parfois à l'encontre du discours politique officiel. Ainsi, dès les années 1990, en désaccord avec la politique environnementaliste, les éleveurs ont reçu de nouveaux prêts pour rénover leurs pâturages dégradés. Derrière cet appui de l'Etat, on retrouve les acteurs de la filière intervenant sur les politiques publiques au niveau local, régional et national.

\section{Valeurs sociales du cheptel}

L'expansion s'explique également par des raisons d'ordre sociologique. En plus d'être un outil de production, le troupeau est une épargne rapidement mobilisable. En cas de problèmes, familiaux par exemple, une partie des animaux peut être vendue pour subvenir aux besoins.

La propriété d'un grand troupeau assure aussi un statut social. Il témoigne de la réussite de l'exploitation et des valeurs pionnières de son propriétaire. Ce mythe du fazendeiro est entretenu par la médiatisation des succès d'ouvriers agricoles ou de petits fermiers devenus de grands 
exploitants à force d'ambition, de labeur et parfois d'absence de scrupules.

Revenir aujourd'hui sur ces facteurs d'engouement des acteurs pour l'élevage est pertinent pour diverses raisons. La première est l'arrivée aux affaires de la première génération de colons née en Amazonie, avec les problèmes de transmission des biens et des savoirs. La deuxième est liée à l'évolution du cadre de vie depuis 20-30 ans, notamment l'amélioration de l'habitat et de l'accès aux services de santé, d'éducation et de retraite. La dernière est l'apparition de nouvelles exigences dans le domaine de l'environnement, du travail et du bien-être.

\section{Une modélisation}

\section{focalisée}

\section{sur les producteurs}

\section{Principales orientations des modèles sur la déforestation amazonienne}

Dans une revue des modèles qui traitent de la déforestation des grandes forêts tropicales, Kaimowitz et Angelsen (1998) montrent que la plupart des modèles disponibles sont fondés sur une évaluation statistique du rôle de différents facteurs à des niveaux macroscopiques, allant jusqu'à représenter tout le bassin amazonien. Souvent conçus à partir d'estimations des surfaces déboisées par télédétection et d'analyses multicritères de facteurs potentiels de la déforestation (prix des intrants et des productions, accessibilité des marchés, densité de population, etc.), ils s'appuient sur une extrapolation des tendances observées pour en prospecter le futur selon des hypothèses optimistes ou pessimistes. Ces modèles comportent deux grandes catégories (Piketty, 2003). Les premiers reposent sur l'identification de la cause principale de la déforestation pour ébaucher les scénarios. Les seconds prennent en compte plusieurs facteurs. Avec ces outils, Nepstad et al. (2001), par exemple, ont construit des scénarios fondés sur une estimation des probabilités de création de nouvelles routes ou d'asphaltage des pistes. Ils ont ainsi estimé qu'au cours des deux décennies suivantes, la déforestation atteindrait de 120000 à 270000 kilomètres carrés, ce qui les a amenés à critiquer le programme "Avança Brasil" (6 000 kilomètres de nouvelles routes). Sans nier l'importance de la route dans le processus, Piketty (2003) note qu'elle constitue souvent le seul facteur explicatif de déforestation pris en compte dans ces modèles, qui font l'hypothèse que ses impacts resteront au même niveau que dans le passé (hypothèse de processus stationnaire). On a pourtant observé des situations où la construction d'une route faisait suite à une déforestation au lieu de la précéder. Si certains estiment qu'un meilleur accès aux routes et aux marchés augmente les probabilités de déforestation, d'autres pensent que la pauvreté, souvent circonscrite dans les zones peu accessibles, est un facteur important de déforestation. Ces modèles fondés sur de simples corrélations proposent donc des explications peu convaincantes et contradictoires des causes de la déforestation. Ils n'offrent qu'un éclairage partiel sur des processus qui restent mal compris. Il est en outre difficile de saisir l'essence des phénomènes étudiés en travaillant à une échelle macrorégionale: comme le montre Lambin (1994), cette dernière occulte la variabilité des situations, en particulier le rôle des comportements sociaux individuels et collectifs. Les acteurs sont représentés dans ces modèles, quand ils le sont, sous forme de densité et de flux de population. Ainsi, en dépit de l'attention prêtée par la communauté internationale à l'Amazonie, les connaissances sur les choix décisionnels des acteurs restent rudimentaires.

\section{Intérêt de la modélisation SMA et attendus du modèle « TransAmazon »}

Parce qu'elle permet de rendre compte des comportements des acteurs (Bousquet et al., 1999), la modélisation SMA paraît un outil pertinent pour révéler certains facteurs décisifs sur la déforestation. Nous l'avons utilisée pour modéliser les pratiques d'utilisation de l'espace par les colons des fronts pionniers à Uruará (commune de la Transamazonienne). Sans nous polariser sur la seule déforestation, nous avons mobilisé à cette fin les résultats d'études impliquant une forte présence sur le terrain et de nombreux entretiens avec les colons pour comprendre l'organisation du travail, leur histoire et leurs réseaux sociaux. L'objectif premier de cette recherche a été de mieux comprendre leurs stratégies, les difficultés qu'ils rencontrent et d'imaginer avec eux des évolutions alternatives.

$\mathrm{Au}$ moyen d'une formalisation des processus naturels et sociaux, la construction du modèle SMA "TransAmazon " a pour objectif de comprendre comment les producteurs utilisent la terre, et quels sont les points clés qui influencent les dynamiques d'utilisation du sol. La modélisation cherche à représenter les activités des petits colons arrivés au début des années 1970 en spécifiant leurs besoins, leurs stratégies de production et leurs interactions, afin de retracer leurs trajectoires d'évolution et d'anticiper les devenirs possibles des fronts pionniers.

\section{Une entrée par le foncier} et l'utilisation du sol

Plusieurs auteurs voient dans la colonisation une vaste opération menée par des groupes d'acteurs individuels et institutionnels. Ianni (1978) et Uztarroz (1990) parlent de "lutte pour la terre ". La déforestation peut être analysée au travers du foncier, car, pour de nombreux producteurs, une parcelle forestière n'est qu'une réserve de fertilité à valoriser. À l'instar du statut qu'offre un troupeau, le foncier véhicule des valeurs positives seulement s'il est mis en production. Si un ranch ou un troupeau valorise son propriétaire dans la société, un lot non défriché fait de lui un fainéant.

En Amazonie, peu d'exploitations sont exclusivement pastorales. Malgré une bonne productivité du pâturage les deux-trois premières années, un exploitant préfère souvent implanter une culture vivrière après le brûlis: elle assure la sécurité alimentaire familiale et son revenu couvre le coût d'implantation du pâturage. Même pour les ranchs, la culture vivrière est l'étape initiale avant pâturage. De plus, la technique la plus pratiquée pour restaurer un pâturage est de le mettre en culture vivrière pendant une ou deux saisons, ce qui constitue ainsi un autre élément de la complémentarité culture-fourrage. Enfin, la raréfaction de la réserve forestière dans les exploitations anciennes oblige certains des premiers pionniers à intensifier l'utilisation du sol par une rotation culture-pâture. Ils cherchent aussi à s'agrandir pour rester sous le seuil légal de déforestation.

\section{Une entrée par la main-d'œuvre}

La disponibilité en main-d'œuvre familiale est un élément clé de la réussite ou 
de l'échec de l'implantation sur un front pionnier. Ferreira (2001) et Hostiou et al. (2006) montrent qu'une famille de troisquatre actifs (un couple avec deux adolescents) a plus de chances de réussir qu'un couple avec de jeunes enfants. La maladie ou l'accident invalidant pendant plusieurs semaines sont moins préoccupants pour une famille de plusieurs actifs. De plus, certaines activités - coupe de bois, déplacement du troupeau, récolte... - requièrent plusieurs personnes. Enfin, la vente occasionnelle d'une part de la force de travail améliore le revenu familial. Ainsi, les cas de réussite sont souvent le fait de familles nombreuses à leur arrivée sur le front, même si elles ont perdu par la suite des enfants par accident ou maladie.

\section{Description de la structure du modèle}

"TranAmazon" est constitué de deux modules correspondant aux paragraphes précédents.

Le module foncier représente l'organisation spatiale. Situé le long d'une route vicinale, chaque lot de colonisation de 100 hectares est divisé en parcelles de 5 hectares. Cette unité de base correspond à la surface moyenne annuellement déforestée par un fermier. Une exploitation peut compter plusieurs lots. À chaque parcelle, est affecté un couvert végétal: forêt, jachère ou culture vivrière, pérenne ou fourragère (figure 1). Une pâture peut contenir un troupeau à raison d'une tête/hectare. Chaque couvert possède des attributs technico-économiques, sa dynamique propre, ses exigences en termes de maind'œeuvre et d'intrants et une production. Le troupeau n'est évidemment pas un couvert végétal, mais la façon dont a été conçue sa dynamique sans prendre en compte le cycle de vie des vaches s'est révélée finalement proche d'une culture : il génère une production (de viande) à chaque saison. Sans entretien, sa productivité diminue et il finit par disparaitre. Ce module spatialisé calcule la production de chaque couvert et le fait évoluer naturellement : sans producteur, un paysage évolue progressivement vers la forêt (figure 2). Pour les échanges fonciers, il calcule la valeur d'un lot qui dépend de sa situation, de la qualité des sols et de la valeur de ses couverts.

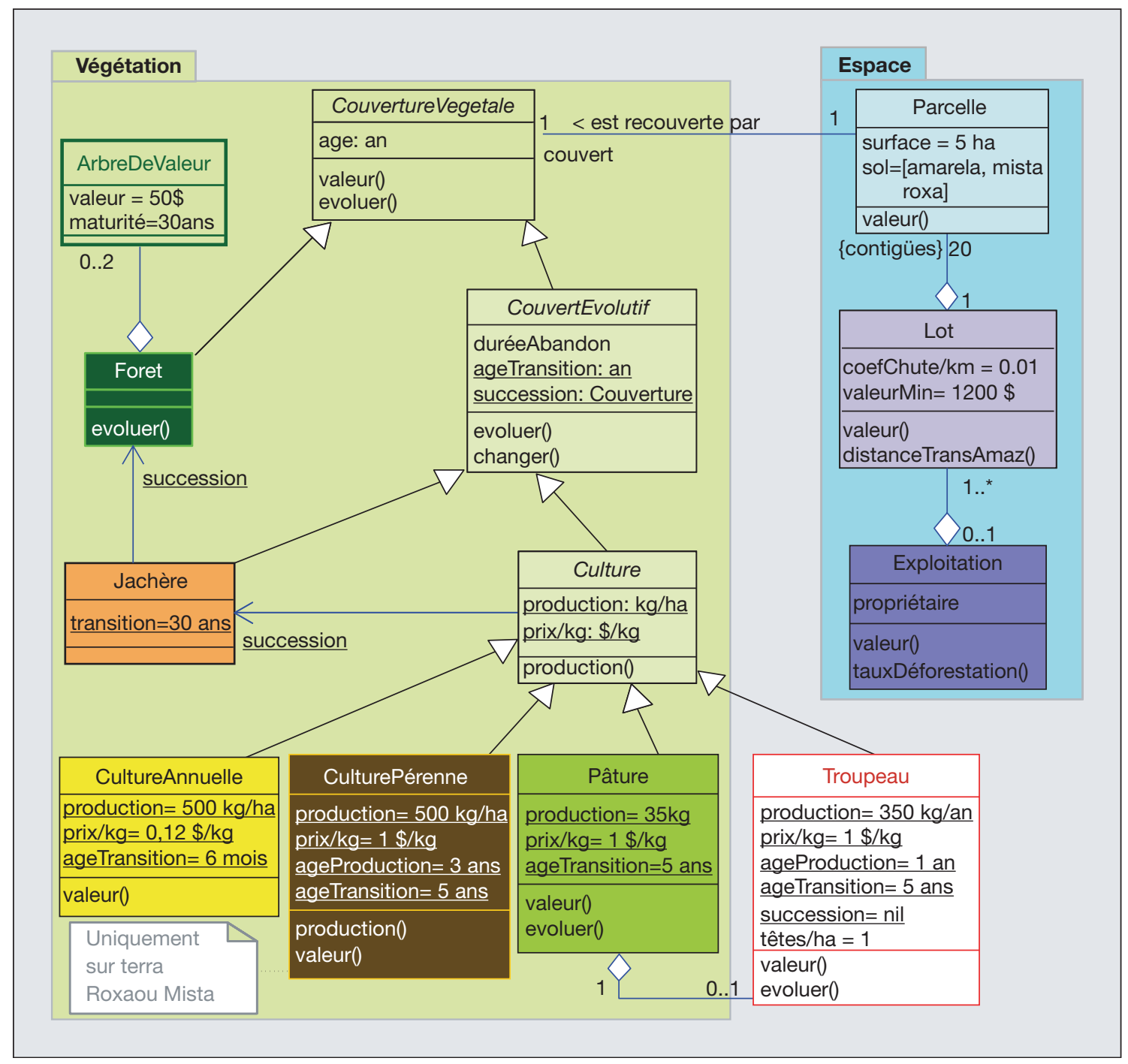

Figure 1. Structure spatiale et couvert végétal (diagramme de classes).

Figure 1. Space structure and vegetal cover (Class Diag.). 


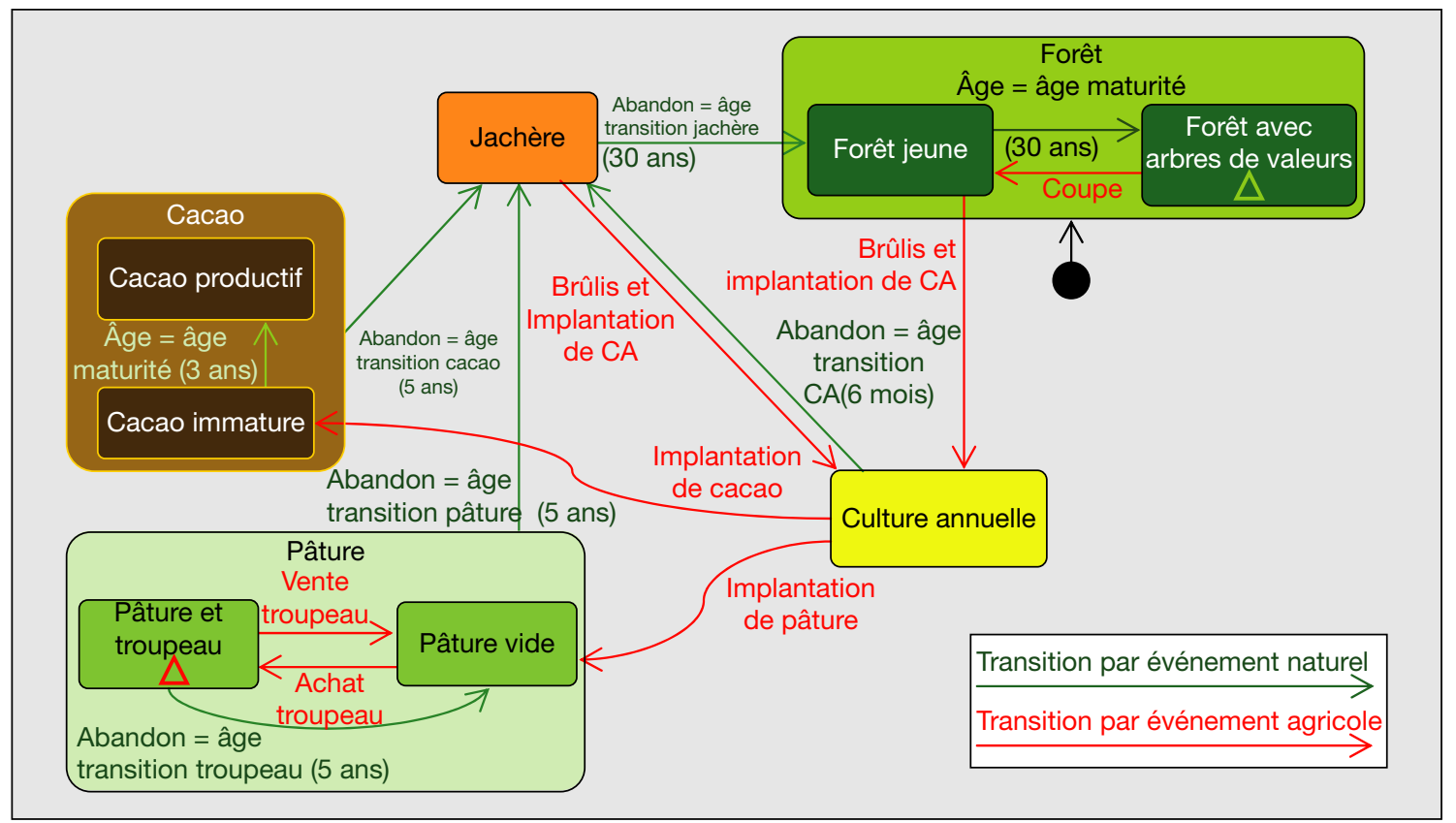

Figure 2. Diagramme d'état-transition de la végétation.

Figure 2. State-transition diagram of vegetation.

Le second module représente la famille. Un agent (une famille de $n$ membres) dispose de deux ressources: sa maind'œuvre (MO), qu'il utilise pour ses travaux ou qu'il vend à l'extérieur, et son argent, qu'il récupère lors des récoltes ou de la vente de sa main-d'œuvre. Il affecte cet argent à la consommation du ménage, aux coûts attachés aux travaux, à l'embauche occasionnelle de main d'œuvre et à l'achat de lots. Sa main-d'œuvre équivaut à une quantité de journées de travail disponible qui diminue avec les tâches effectuées. Cette quantité est réactualisée en début de saison (figure 3).

Une famille est associée à une stratégie qui consiste à réaliser des opérations agricoles en fonction de la saison, sèche ou humide, à vendre ou acheter de la maind'œuvre, à effectuer un bilan annuel pour acheter ou vendre des vaches ou un lot. On différencie les stratégies des agents 1) sans terres, pour les familles sans lot ; 2) planteur ; 3) éleveur, pour les exploitants (figure 4).

En choisissant d'être planteur ou éleveur, une famille cultive sa terre en "privilégiant" sa spécialité. Mais ce choix ne signifie pas forcément un abandon des autres cultures déjà en place sur le lot. En fonction de ses résultats et de ceux des voisins, un agent choisit sa prochaine stratégie. Ce procédé favorise l'apparition de trajectoires évolutives sophistiquées.

\section{Quelques résultats}

Les agents sont uniquement créés en début de simulation. La seule dynamique démographique est liée à l'exclusion des agents trop endettés. Il n'y a ni reproduc- tion ni arrivée de nouveaux colons ce qui limite la durée des simulations à 25 ans. Une simulation standard montre l'évolution du paysage en forme d'arête de poisson typique (figure 5). Outre une représentation spatiale dynamique, elle

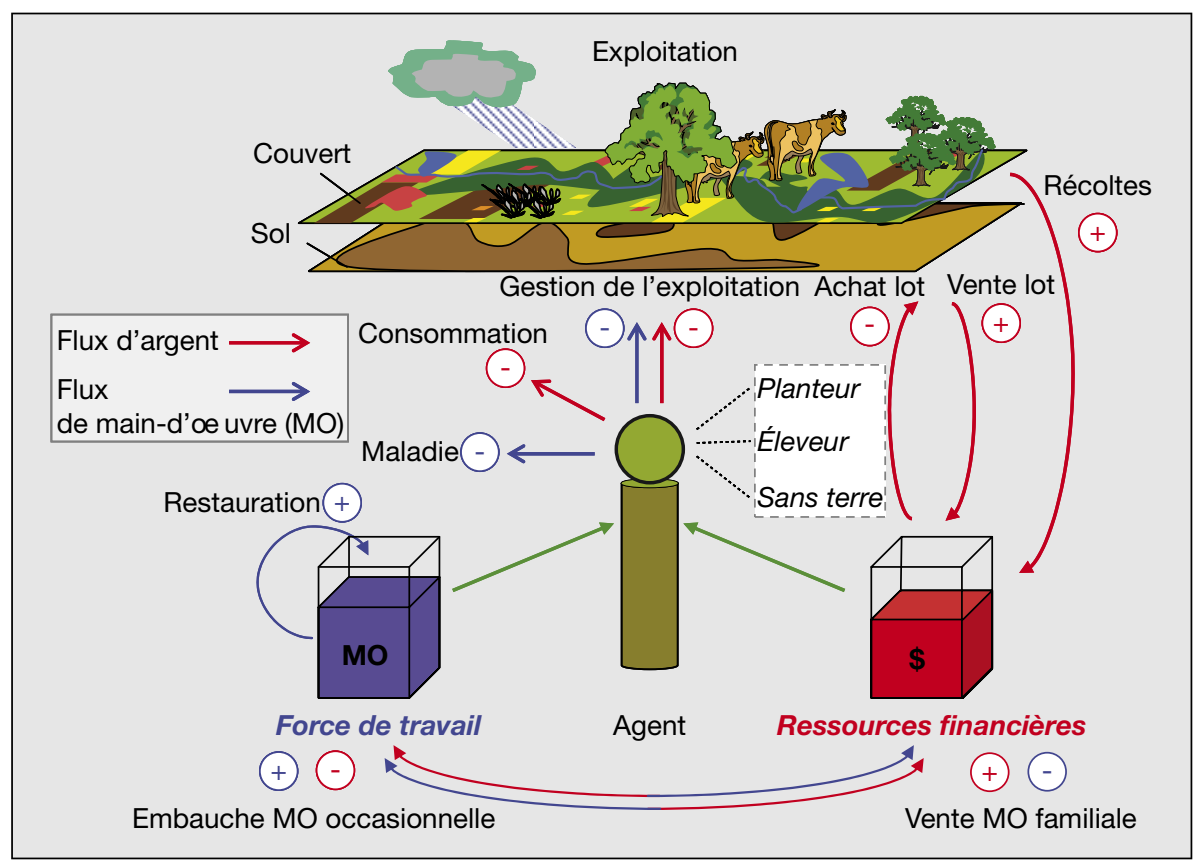

Figure 3. Diagramme systémique d'un agent et de son lot.

Figure 3. Systemic diagram of an agent and farm. 


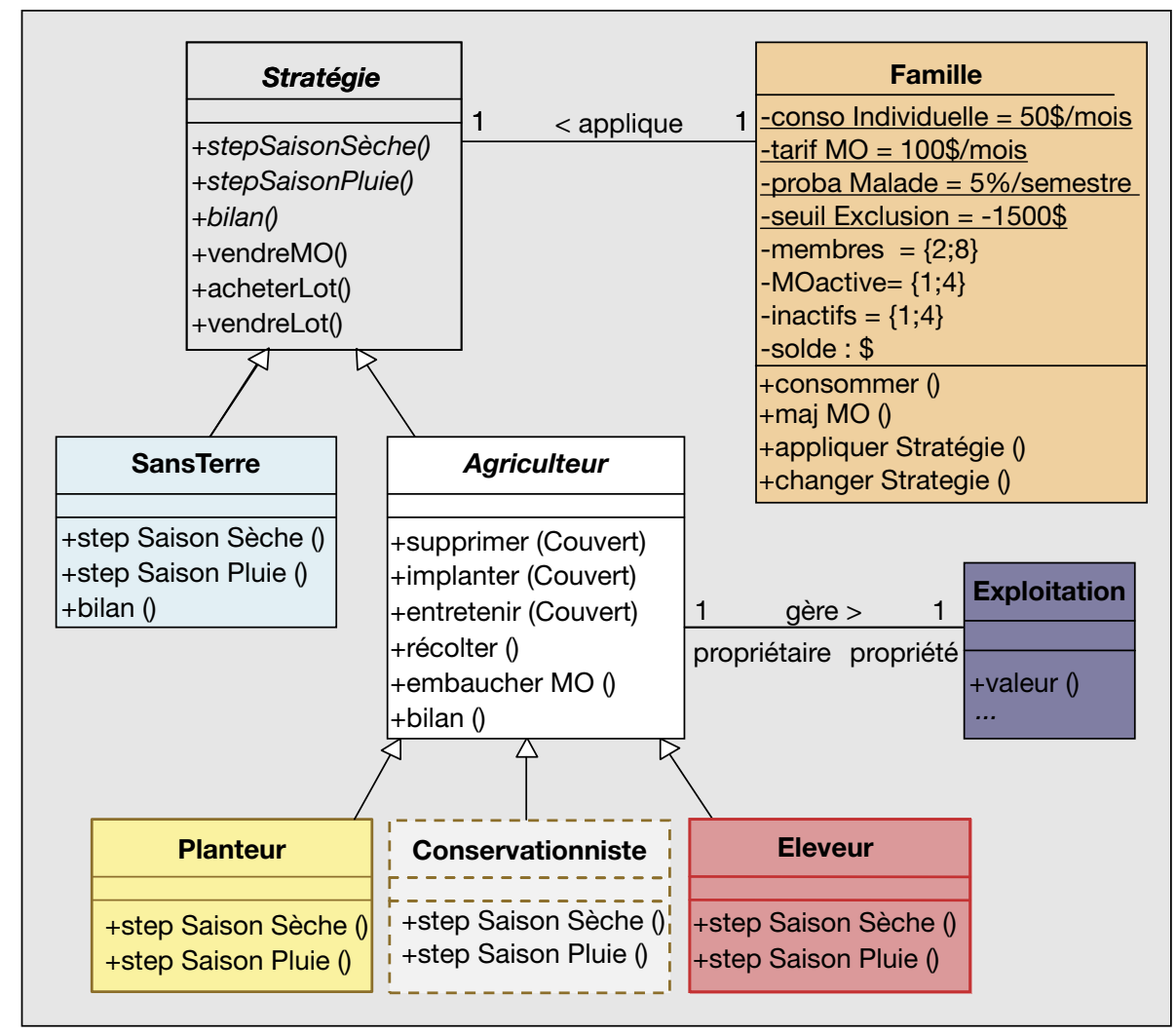

Figure 4. Structure des agents (diagramme de classe). La classe " conservationniste " a été ajoutée ultérieurement.

Figure 4. Structure of the agents. The Conservationist class was added later on.

permet de suivre l'évolution de nombreux indicateurs individuels - couverture d'un lot, capital d'un agent, ses ventes, etc. - ou globaux - nombre d'exclusions, économie de la communauté, surface des forêts.

Même si sa rentabilité est réduite, l'élevage domine : contrairement à la production de cacao, il est rapidement rentable ; ses coûts d'implantation et d'entretien sont faibles; si le bénéfice est limité, le troupeau constitue une garantie face aux risques et la simulation montre des ventes de bétail lors de difficultés financières.

Alors que la déforestation est accentuée pour des prix fluctuants du cacao, une simulation avec prix stable illustre une situation gagnant-gagnant avec une réduction de la déforestation et un meilleur niveau de vie des agents.

D'autres descriptions et résultats sont disponibles $^{2}$ (Bonaudo, 2005; Bonaudo et al., 2005).

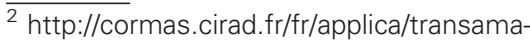
zon.htm.
}

\section{Utilisations et évolutions du modèle}

La version de "TransAmazon " présentée ici ne permet pas de tester des mesures incitatives pour préserver la forêt: les agents ne modifient pas leur pratique en fonction du montant de l'indemnité, car ils n'ont pas d'autres possibilités que de planter ou élever. Il a donc fallu ajouter une stratégie "conservationniste" à la figure 4 qui consiste à seulement entretenir les cultures déjà en place. On peut alors tester des politiques d'incitation en augmentant le revenu issu de la forêt. Ici aussi, les résultats sont surprenants : une indemnité inférieure à 15 dollars US/hectare de forêt serait pire que de ne rien faire. À partir de 18 dollars US/hectare, la déforestation s'arrête, mais seulement après une période de 10 ans qui s'avère redoutable. À partir de 21 dollars US/hectare, la politique semble efficace et il n'est pas nécessaire de monter au-delà pour espérer encore mieux (figure 6). Ces valeurs sont cohérentes avec celles qui sont proposées par Vosti et al. (2002) et Cattaneo (2002).

Lors du bilan annuel, l'agent change de stratégie en comparant ses revenus passés avec ceux de ses voisins, mais il ne peut pas anticiper. Une autre version du modèle propose des agents prospectifs qui explorent virtuellement les stratégies disponibles avant de choisir la plus rentable. Les possibilités d'assolement sont limitées par les stratégies actuelles, cohérentes avec nos observations, mais qui n'autorisent pas l'innovation. Afin d'éviter des ajouts $a d$ hoc à chaque mesure incitative à tester, nous développons une autre version qui permettra d'augmenter les capacités d'adaptation des agents : les stratégies préétablies y sont éliminées et l'agent imagine des combinaisons d'activités élémentaires (défricher, implanter, entretenir...) dont chacune consomme du travail et de l'argent. En explorant un ensemble d'itinéraires, il construit dynamiquement sa stratégie. En fondant les décisions sur une minimisation des risques, on espère retrouver les stratégies prédéfinies, mais aussi de nouveaux itinéraires plus efficaces.

D'un modèle descriptif présentant des comportements stéréotypés, "TransAmazon" devient ainsi un modèle plus générique dans lequel la gestion du risque prend une dimension jusqu'alors sous-estimée.

Pour freiner l'avancée des fronts pionniers, des techniques et des pratiques adaptées au contexte amazonien ont été imaginées. Elles furent formalisées par un modèle graphique d'intensification agricole, RPLB (Roça permanente e Lavoura Branca), adapté de "TransAmazon" à l'occasion de rencontres avec des paysans, des techniciens et des gestionnaires de ranchs. Cela a conduit à des expérimentations de ces nouvelles pratiques. L'amélioration du modèle RPLB se poursuit ainsi selon deux axes : la gestion des parcelles forestières et la récupération des aires protégées (berges, bas-fonds, pentes). En intégrant ces aspects, une version interactive de "TransAmazon " permettra au producteur d'interagir avec son exploitation virtuelle pour imaginer et tester de nouveaux itinéraires techniques.

\section{Enseignements tirés de la modélisation}

Les nombreuses recherches antérieures sur nos terrains ont joué en faveur d'une modélisation à dires d'experts. Mais nos travaux s'appuient sur des entretiens, des enquêtes et des expérimentations en milieu paysan. Durant ces opérations, le modèle fut discuté, amendé et approuvé. Une modélisation participative associant 


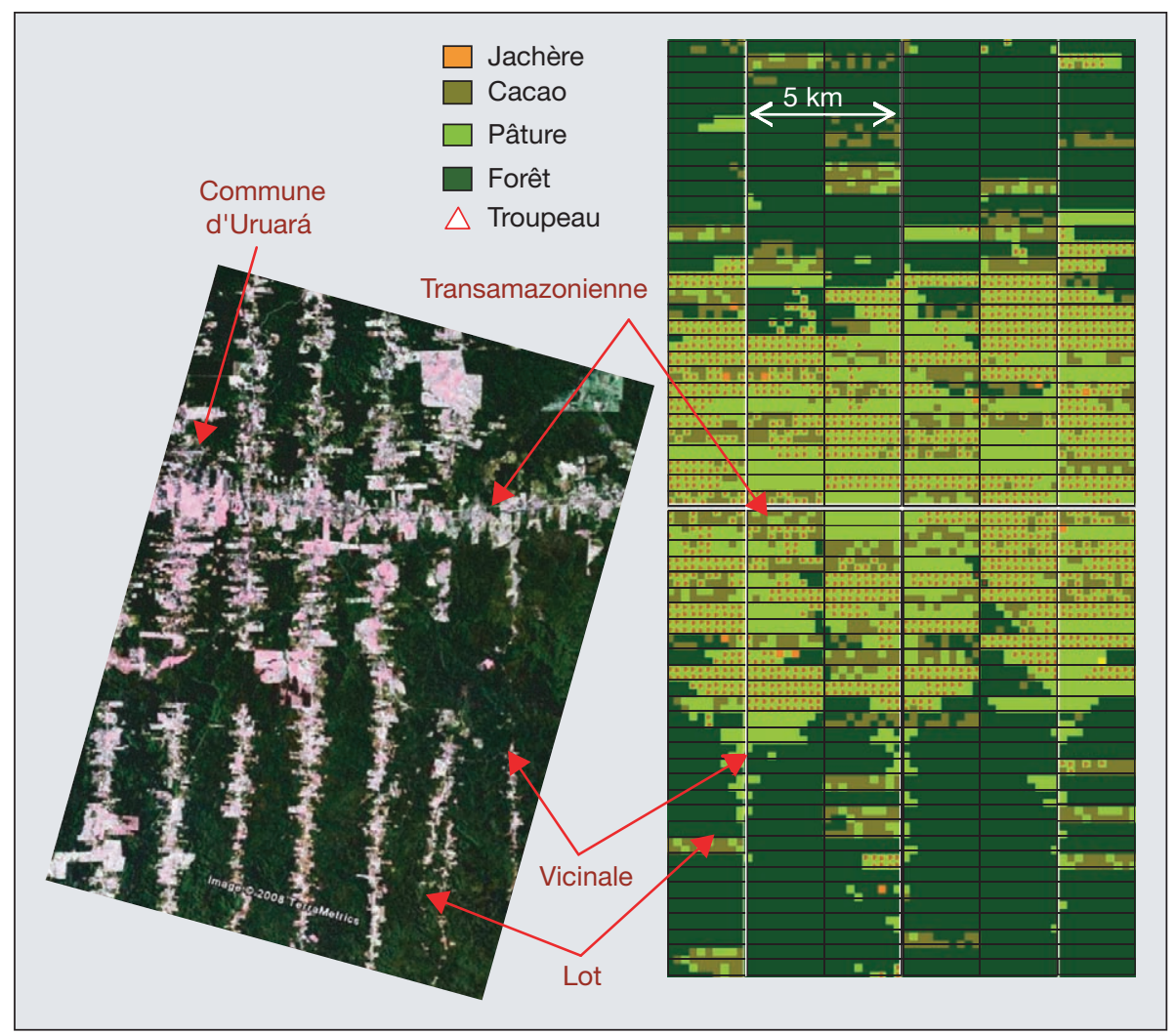

Figure 5. La déforestation sur la Transamazonienne, photo et simulation.

Figure 5. The deforestation of the Transamazonian road: A photo and a view of a simulation. À gauche, photo satellite de la Transamazonienne (source Google Earth) montrant la structure en arête de poisson ; à droite, vue d'une simulation.

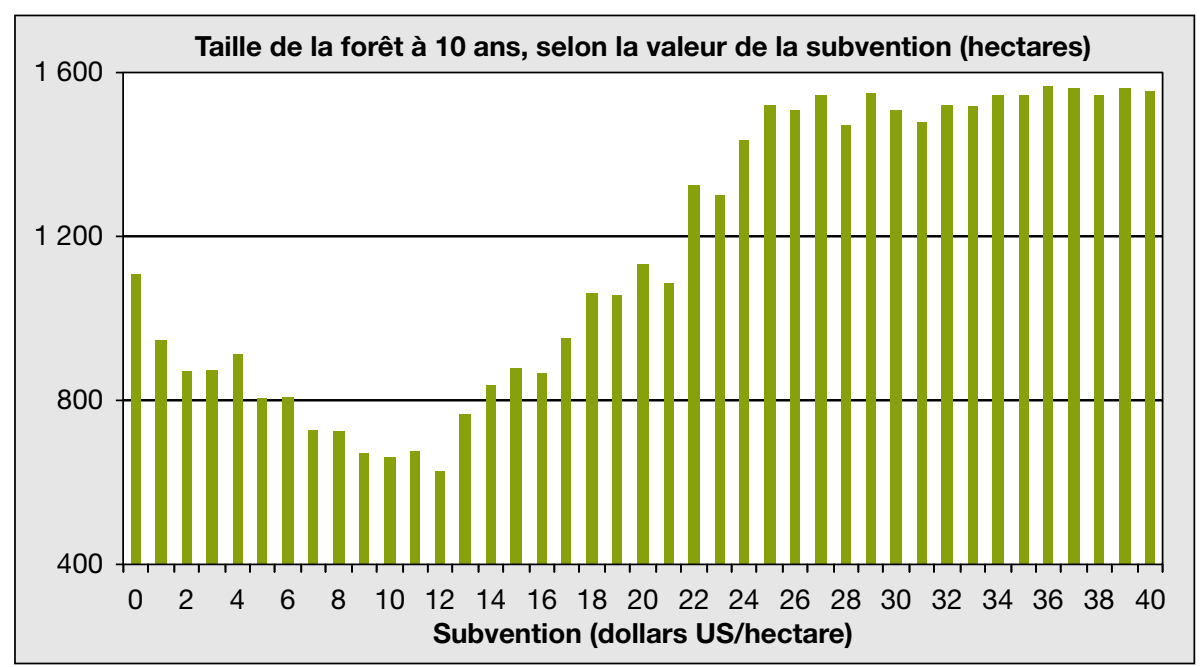

Figure 6. Analyse d'une politique de subvention pour préservation de la forêt: superficie de la forêt à 10 ans selon le niveau de la subvention.

Figure 6. Analysis of a forest preservation policy: forest area at 10 years according to the level of the subsidy. les acteurs locaux dès le début aurait été plus longue et onéreuse.

Le premier enseignement a été le partage de savoir entre les différents experts et leurs réseaux d'acteurs pour arriver à une vision commune sur les éléments du modèle.

Le deuxième enseignement est l'intérêt de simplifier: en partant d'une typologie reconnue (Ferreira, 2001), la modélisation n'a retenu que trois stratégies, dont la combinaison dans le temps permet de retrouver les sept types initiaux. Ainsi, des aspects importants pour les experts ne figurent pas explicitement dans le modèle. Il s'agit d'une montée en abstraction vers un modèle plus générique.

Le troisième enseignement est l'apport de la modélisation à la transdisciplinarité : la diagrammatique utilisée oblige à formaliser les concepts et à mieux les décrire. Ainsi, les experts en sciences sociales doivent comprendre ce que les techniciens ont mis dans les composantes techniques et vice versa. Les supports graphiques facilitent cette collaboration et l'exploration collective des simulations oblige à des remises en cause constructives. Si les débats entre modélisateurs et thématiciens furent intenses, autant que ceux entre acteurs locaux, ils favorisèrent l'évolution des concepts.

\section{Conclusion}

À l'opposé des macromodèles sur l'Amazonie, "TransAmazon" se fonde sur une approche des pratiques d'utilisation de l'espace par des familles de colons, principaux acteurs de la déforestation. Il permet de retrouver les schémas généraux de la déforestation, non pas par de simples corrélations liées à la présence de routes ou de marchés, mais à partir du comportement des acteurs et de l'impact des pratiques agricoles sur l'espace. De plus, il permet de tester les conséquences de politiques publiques sur le développement durable. Néanmoins, le modèle n'a pas vocation à être un outil au service des seuls décideurs. En nous centrant sur les stratégies de gestion de l'espace des colons, nous avons cherché avant tout à discuter avec eux de leurs pratiques et de leurs modes d'organisation dans une optique d'appui au développement.

Notre objectif est maintenant de faire évoluer le modèle vers un outil de pilotage à l'échelle locale que les acteurs individuels et collectifs pourraient s'approprier afin 
de tester de nouvelles stratégies de gestion et d'élaborer des politiques plus respectueuses des ressources forestières.

\section{Références}

Bonaudo T. La gestion environnementale sur un front pionnier amazonien. Thèse de doctorat de I'INA-PG, Paris, 2005. http://pastel. paristech.org/3472/

Bonaudo T. Bommel P. Tourrand JF. In: Modelling the Pioneers Fronts of the transamazonhighway region. Bourg St Maurice (France): Smaget, Multi-Agent Modelling for Environmental Management, 2005.

Bousquet F, Barreteau O, Le Page C, Mullon C, Weber J. An environmental modelling approach: the use of multi-agent simulations. Advances in environmental and ecological modelling. Paris : Elsevier, 1999.

Cattaneo A. Balancing agricultural development and deforestation in the Brazilian Amazon. Washington DC: International Food Policy Research Institute (IFPRI), 2002.
Ferreira LA. Le rôle de l'élevage bovin dans la viabilité agro-écologique et socio-économique des systèmes de production agricoles familiaux en Amazonie brésilienne - le cas d'Uruará (Pará, Brésil). Thèse de doctorat, INA-PG, 2001. http:// www.cultura.ufpa.br/cagro/pdfs/AA_Agriculturas_Amazonicas/T_LAURA_FERREIRA.pdf

Hostiou N, Tourrand JF, Veiga JB, Negreiros Alves AM, Barbosa T. The labour organization of small-scale breeders in the Brazilian Amazon is a key point for sustainable development. In: Changing European farming systems for a better future. New visions for rural areas. IFSA Symposium 7. Wageningen, Pays-Bas. 2006 Wageningen : Academic Publishers, 2006.

Kaimowitz D et Angelsen A. Economic models of tropical deforestation, a review. Revue du CIFOR 1998: 153. http://www.cifor.cgiar.org/ publications/pdf_files/Books/model.pdf

Ianni O. A luta pela tera. Petrópolis-RJ : Vozes Ltda 1978

Piketty MG. Mudancas do uso da terra e da floresta e efeito estufa nas frentes pioneiras da Amazônia Brasileira. São Paulo : Université de São Paulo, 2003. http://agents.cirad.fr/index. php/Marie-Gabrielle+PIKETTY/Publications
Piketty MG, Veiga JB, Tourrand JF, Negreiros Alves AM, Poccard Chapuis R, Thales MC. Les déterminants de l'expansion de l'élevage bovin en Amazonie orientale : conséquences pour les politiques publiques. Cah Agric $2005 ; 14: 90-5$.

Poccard Chapuis R, Thales MC, Venturieri AP, et al. La filière viande : un levier pour contrôler les dynamiques pionnières en Amazonie brésilienne ? Cah Agric 2005; $14: 53-8$.

Toni F. State-Society Relations on the Agricultural Frontier: the Struggle for Credit in the Transamazônica Region. PhD, université de Florida, Gainesville, USA, 1999.

Uztarroz R. L'Amazonie, la foire d'empoigne. Série Monde HS, $n^{\circ} 49$ Paris : Autrement, 1990.

Veiga JB, Tourrand JF, Piketty MG, PoccardChapuis R, Alves AM, Thales MC. Expansão e trajetórias da pecuária na Amazônia: Estado do Pará. Brasilia : Editora da Universidade de Brasília, 2004.

Vosti SA, Witcover J, Carpentier CL. Agricultural Intensification by Smallholders in the Western Brazilian Amazon: From Deforestation to Sustainable Land Use. Washington (DC) : International food policy research institute, 2002. 